\title{
CHANGES IN BACKGROUND ELECTROENCEPHALOGRAPHIC ACTIVITY IN PATIENTS WITH EPILEPSY AND/WITHOUT COMORBID DEPRESSION
}

\author{
Stefan Tsekov ${ }^{1}$, Koraliya Todorova ${ }^{2}$ \\ ${ }^{1}$ Medical College, Medical University of Varna, ${ }^{2}$ First Clinic of Psychiatry, \\ St. Marina University Hospital of Varna
}

\begin{abstract}
PURPOSE: Depressive disorders are the most common interictal psychiatric comorbidity in epilepsy. Recent data highlights the possibility of a close bi-directional pathophysiological relationship between these two disorders. Early detection and treatment of interictal depressive disorder (DD) may significantly improve patients' quality of life. The aim of the present study was to compare recorded interictal electroencephalographic (EEG) data in patients with epilepsy with and without comorbid DD.

MATERIAL AND METHODS: Forty patients with epilepsy were divided into two groups according to the presence/absence of comorbid DD. They were diagnosed by applying the International Classification of Diseases, Version 10 (ICD-10) diagnostic criteria. Evaluation on Hamilton Depression Rating Scale (HAM-D-17) supported the diagnosis. Nineteen-electrode electroencephalography (EEG) with spectral analysis of the power was recorded for each participant. Gender- and age-matched subjects comprised a control group.

RESULTS: An increase in slow wave EEG activity in the frontal and temporal lobe as well as a decrease in sensory motor rhythm was found out in the group of patients with epilepsy and comorbid DD. No such findings were detected in the group of patients with epilepsy only.

CONCLUSION: Atypical presentation of interictal DD is common in epilepsy. Comorbid depression remains unrecognized and untreated in a great proportion of patients. Detection of certain patterns of EEG changes may be useful as a screening tool for forwarding these patients to a further psychiatric evaluation. It can serve as a basis for applying alternative therapeutic methods such as the biofeedback method.
\end{abstract}

Key words: epilepsy, interictal depressive disorder, electroencephalographic activity, Hamilton Depression Rating Scale

"Melancholics ordinarily become epileptics, and epileptics melancholics: what determines the preference is the direction the malady takes; if it bears upon the body, epilepsy, if upon the intelligence, melancholy."

Hippocrates (15)

Address for correspondence:

Stefan Tsekov, MD, PhD

Medical College, Medical University of Varna,

84, Tzar Osvoboditel Blvd., 9000 Varna, Bulgaria

Mobile: 00359889464 254;

e-mail:stefan.tsekov@gmail.com

Received: August 30, 2012

Accepted: January 15, 2013

\section{INTRODUCTION}

Depressive disorders (DD) are the most common interictal psychiatric comorbidity in epilepsy with a lifetime prevalence of about 30$60 \%(1,20)$. There is no simple explanation of the pathophysiological relations between epilepsy and DD. Common pathogenic mechanisms mediated by a neurotransmitter dysfunction or relation to 
the location of the seizure foci, type of epilepsy or reaction to the psychosocial implications of living with epilepsy, respectively, could matter (11-14,18). Attempts to treat seizures without considering the presence of a comorbid DD are equivalent to an incomplete treatment of these patients (14). Drug interactions, medication side effects and financial concerns may be barriers to the administration of antidepressant medications to the patients requiring long term antiepileptic drug therapy. Biofeedback is an encouraging development that holds promise as a method for modifying biological brain patterns associated with a variety of psychiatric and general medical conditions. It is a non-invasive therapeutic option rarely associated with even mild side effects which offers an alternative to the invasive ones such as electroconvulsive therapy and intense transcranial magnetic stimulation (9). Biofeedback training may be presented as a possible treatment strategy for patients with epilepsy and certain comorbid conditions, including DD.

The purpose of this study was to evaluate and compare recorded interictal quantitative electroencephalographic (EEG) data in patients with epilepsy with or without comorbid DD focusing on the EEG parameters which could contribute to their complex diagnosis and treatment.

\section{MATERIAL AND METHODS}

Forty outpatients ( 15 males and 25 females aged 18 to 52 years) with a confirmed diagnosis of epilepsy based on documented clinical data and prolonged EEG monitoring and with complex partial seizures with or without secondary generalization lasting at least 2 years were equally divided into two groups according to the presence/absence of comorbid DD. Exclusion criteria were: history of alcohol or substance abuse, encephalopathy or progressive structural brain abnormality documented by a computed axial tomography (CAT) or magnetic resonance tomography (MRT), severe comorbid somatic disease, treatment with benzodiazepines (e. g. clonazepam) and barbiturates. The International league against epilepsy (ILAE) clinical and EEG classification of epileptic seizures and epilepsies was applied (2). Comorbid DD was diagnosed according to the International Classification of Diseases, Version 10 (ICD-10) diagnostic criteria (21). The severity of the depression was evaluated by Hamilton Depression Rating Scale (HAM-D-17) (8). Forty gender- and age-matched controls were chosen for each patient with epilepsy.

Nineteen-electrode EEG was recorded for all the participants applying the international 1020 coordinate system for electrode placement. The quantitative EEG (qEEG) measurement included the spectral analysis of the absolute power $(\mu \mathrm{V} 2 / \mathrm{Hz})$ in theta- 5 to $8 \mathrm{~Hz}$, alpha- 8 to $12 \mathrm{~Hz}$, and beta- 16 to 2 of the background activity $5 \mathrm{~Hz}$ bands as well as the absolute power of the sensory motor rhythm (SMR) - 12 to $15 \mathrm{~Hz}$.

Statistical analysis included Student's $t$-test.

\section{RESULTS}

An increase of the slow wave EEG activity $(\mathrm{p}<0,01)$ in the frontal and temporal lobe as well as a decrease of the absolute SMR power $(\mathrm{p}<0,001)$ and beta band $(\mathrm{p}<0,001)$ was found out in the group of patients with epilepsy and comorbid DD compared to the patients with epilepsy only (Fig. 1).

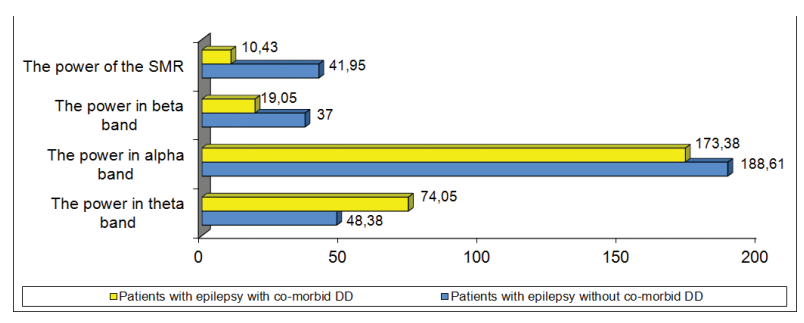

Fig. 1. Comparative analysis of the qEEG data for the patients with epilepsy with/without comorbid DD

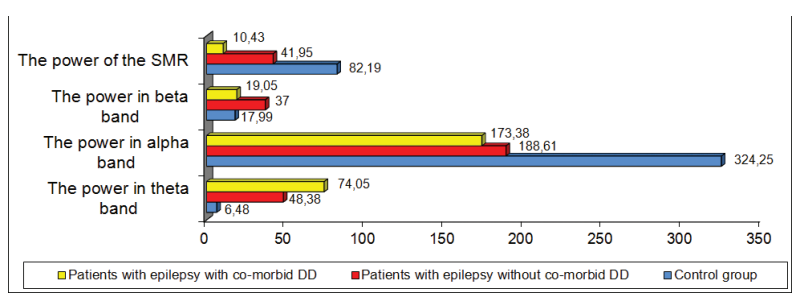

Fig. 2. Comparative analysis of the qEEG data for the patients with epilepsy with/without comorbid DD and the control group 
Similar changes regarding the theta, alpha, beta bands as well as SMR power were established in both groups compared to the control subjects (Fig. 2).

\section{DISCUSSION}

Previous research indicates a significant correlation between the type and frequency of focal seizures, anticonvulsive drugs, psychosocial factors and incidence of comorbid DD (7). DD may be diagnosed at any time during the course of epilepsy and a history of depression preceding the onset of a seizure disorder is more frequent among patients with epilepsy than in a control group (14). Making the diagnosis is the most important step in appropriately managing mood disorders in the epilepsy population (3). Screening tools for rapid detection of comorbid depression in epilepsy such as the Neurological Disorders Depression Inventory for Epilepsy (NDDI-E) that has recently been created and validated should be more widely used by neurologists in clinical settings (6).

Our results show specific qEEG differences in the group of patients with epilepsy and comorbid DD compared to the patients with epilepsy only. On the contrary, Robertson et al. failed to establish any differences in the EEGs of the patients with epilepsy and comorbid DD and a control group (17). Hughes et al. evaluated EEGs of psychiatric patients with major depression and reported specific changes in the absolute power in the alpha band compared to the findings in a control group (10). Our results about absent specific differences in the absolute alpha power for both study groups, with epilepsy and comorbid DD and epilepsy only, allow us to believe that the changes in the absolute power of the alpha band in patients with epilepsy should not be regarded as an indicator for potential DD comorbidity. They are in agreement with recent observations that biofeedback using a combination of SMR enhancement with suppression of slow wave activity can be effective in reducing seizure frequency $(5,16,19)$. One should pay attention to the considerations that the changes of the beta activity could play a role in the treatment of epilepsy and depression (4).

The question of the scientific evidence suggesting a bidirectional electrophysiological interaction between epilepsy and depression should be highlighted in future research and verified in clinical practice.

\section{CONCLUSION}

Detection of certain qEEG changes may be useful as a screening tool for forwarding the epilepsy patients to a further psychiatric evaluation. These patterns may serve for applying alternative therapeutic methods such as the biofeedback method.

Suggestions that biofeedback of slow direct current potentials could be effective in reducing both seizure frequency and frequency and/or severity of comorbid DD in epilepsy patients should be taken into consideration. Moreover, higher SMR and beta activity as well may play a significant role in the treatment of these two medical conditions, i.e. epilepsy and comorbid DD.

\section{REFERENCES}

1. Beyenburg, S., A. J. Mitchell, D. Schmidt, C. E. Elger, M. Reuber. Anxiety in patients with epilepsy: systematic review and suggestions for clinical management.- Epilepsy Behav., 7, 2005, No 2, 161-171.

2. Commission on classification and terminology of the International league against epilepsy (ILAE). Proposal for revised clinical and electroencephalographic classification of epileptic seizures.- Epilepsia, 22, 1981, No 4, 489-501.

3. de Toffol, B., P. Corcia, J. Praline, K. Mondon. The burden of psychiatric comorbidity in epilepsy.Epilepsia, 19, 2007, No 4, 231-241.

4. Dumermuth, G., T. Gasser, P. Germann, A. Hecker, M. Herdan, B. Lange. Studies on EEG activities in the beta band. - Eur. Neurol., 16, 1977, No 1-6, 197-202.

5. Finley, W. W., H. A. Smith, M. D. Etherton. Reduction of seizures and normalization of the EEG in severe epileptic following sensorimotor biofeedback training: preliminary study.- Biol. Psychol., 2, 1975, No 3, 189-203.

6. Gilliam, F. G., I. J. Barry, B. P. Hermann, K. J. Meador, V. Vahle, A. M. Kanner. A rapid detection of major depression in epilepsy: a multicenter study.- Lancet Neurol., 5, 2006, No 5, 399-405. 
7. Grabowska-Grzyb, A., J. Jedrzejczak, E. Naganska, U. Fiszer. Risk factors for depression in patients with epilepsy.- Epilepsy Behav., 8, 2006, No 2, 411-417.

8. Hamilton, M. A rating scale for depression.- J. Neurol. Neurosurg. Psychiatry, 23, 1960, 56-62.

9. Hammond, D. C. Neurofeedback treatment of depression and anxiety.- J. Adult Dev., 12, 2005, No 2-3, 131-137.

10. Hughes, J. R., E. R. John. Conventional and quantitative electroencephalography in psychiatry.- J. Neuropsychiatry Clin. Neurosci., 11, 1999, No 2, 190-208.

11. Indaco, A., P. B. Carrieri, C. Nappi, S. Gentile, S. Striano. Interictal depression in epilepsy.- Epilepsy Res., 12, 1992, No 1, 45-50.

12. Jobe, P. C. Common pathogenic mechanisms between depression and epilepsy: an experimental perspective.- Epilepsy Behav., 4, 2003, Suppl. 3, S14-S24.

13. Kanner, A. M. Epilepsy and mood disorders.Epilepsia, 48, 2007, Suppl. 9, 20-22.

14. Kanner, A. M., A. Balabanov. Depression and epilepsy: how closely related are they?- Neurology, 58, 2002, No 8, Suppl. 5, S27-S39.

15. Lewis, A. Melancholia: a historical review.- J. Ment. Sci., 80, 1934, 1-42.
16. Lubar, J. F., W. W. Bahler. Behavioural management of epileptic seizures following EEG biofeedback training of the sensory motor rhythm.- Biofeedback Self Regul., 1, 1976, No 1, 77-104.

17. Robertson, M. M., M. R. Trimble, H. R. Townsend. Phenomenology of depression in epilepsy.Epilepsia, 28, 1987, No 4, 364-372.

18. Shamin, S., G. Hasler, C. Liew, S. Sato, W. H. Theodore. Temporal lobe epilepsy, depression and hippocampal volume.- Epilepsia, 50, 2009, No 5, 1067-1071.

19. Sterman, M. B., L. Friar. Suppression of seizures in an epileptic following sensorimotor EEG feedback training.- Electroencephalogr. Clin. Neurophysiol., 33, 1972, No 1, 89-95.

20. Tellez-Zenteno, J. F., S. B. Patten, N. Jetté, J. Williams, S. Wiebe. Psychiatric comorbidity in epilepsy: a population based analysis.- Epilepsia, 48, 2007, No 12, 2336-2344.

21. World Health Organization. The ICD-10 Classification of Mental and Behavioural Disorders. Clinical Descriptions and Diagnostic Guidelines. Geneva. WHO, 1992. 